\title{
Cryptocurrencies in Portfolios: Return-liquidity trade-off around China forbidding initial coin offerings
}

Sijia Zhang and Andros Gregoriou

All authors are from Brighton Business School, University of Brighton, Mithras House, Lewes Road, Brighton. BA2 4NT, UK.

\section{$\underline{\text { Abstract }}$}

We examine diversification when cryptocurrencies are included in investment portfolios, around China prohibiting initial coin offerings on the $4^{\text {th }}$ September 2017 . We discover once we account for liquidity, all portfolio diversification benefits of cryptocurrencies are eliminated.

JEL Classification: G11; G14

Key words: Cryptocurrency; Liquidity; Portfolio returns; Bid-ask spread; Amihud ratio

* Corresponding author a.gregoriou@brighton.ac.uk 


\section{Introduction}

Since Bitcoin's inception in 2008 by Nakamoto, the considerable growing cryptocurrencies have received significant attention from market participants, researchers and regulators. According to coinmarketcap.com, by 28 Jan 2020, the total number of cryptocurrencies has surpassed 5,000, the total market capitalization is more than 247 billion USD, and the trading volume has jumped to 114 billion USD. Cryptocurrencies are free from any central bank intervention and have low transaction fees, returns are internally driven by buyers and sellers and are not influenced by fundamental economic factors (Briere et al., 2015; Baek and Elbeck, 2015). One of the reasons that cryptocurrencies are extremely popular is their low correlations with both traditional financial assets and alternative investments. This is why including cryptocurrencies in portfolio diversification strategies greatly reduces the overall risk of the investment (Guesmi et al., 2019). Therefore, cryptocurrencies deliver exceptional high diversification benefits.

The investment in cryptocurrencies exhibits very high volatility however, investors are compensated for this high risk through the extremely low correlations with other assets. As a result of this, including cryptocurrencies into a diversified portfolio is highly profitable (Bouri et al., 2017). Corbet et al. (2018) indicate that the cryptocurrencies obtain effective diversification ability particularly for short-term investors. Gregoriou (2019) concludes that including cryptocurrencies can improve the overall performance of the portfolios. This is because investors can earn excess returns by speculating in cryptocurrencies. Guesmi et al. (2019) examine the most popular cryptocurrency, Bitcoin, and find that Bitcoin is a reliable tool which can diversify the risk of different financial assets, including equities. On the other hand, Briere et al. (2015) discover that a small share of cryptocurrencies such as Bitcoin may lead to substantial financial gains, but simultaneously to a significant increase in extreme risk. It can seriously harm the investor's financial position. Bouri et al. (2017) report that Bitcoin is 
not good for risk management and is suitable for diversification purposes only. Platanakis et al (2018) compare the performance of a naïve and optimal weekly rebalancing of cryptocurrency portfolios. They find that there is no significant performance difference between the different methods of portfolio diversification. However, Platanakis and Urquhart (2019a) discover that sophisticated portfolio techniques, which control for estimation errors are preferred when managing cryptocurrency portfolios. Platanakis and Urquhart (2019b) report that investors should include Bitcoin in their portfolio as it generates substantial higher risk-adjusted returns.

We contribute to the previous literature in the following two ways. First, we are the only study to examine cryptocurrencies' liquidity influence on portfolio performance. Given that cryptocurrencies are excessively volatile, liquidity is a fundamental factor for investors to consider when they include cryptocurrencies in their investment portfolios.

Our second contribution is that we examine the liquidity of cryptocurrencies in an investment portfolio around one of the most significant negative news namely, the Chinese government banning initial coin offerings (hereafter, ICOs). On the $4^{\text {th }}$ of September 2017, several Chinese central financial institutions jointly announced the prohibiting of all cryptocurrency trading activities. China was one of the most important cryptocurrency trading nations in the world. For instance, in 2016 almost $100 \%$ of Bitcoin was trading in China. Zhang and Gregoriou (2020) measure the impact of liquidity over the same event period. They find that China banning ICOs has a negative influence on cryptocurrencies' performance. This is indicating by the trading price and return decreasing, whilst the bid-ask spread increases. Given that China banning ICOs has decreased the liquidity in the market, we establish if the liquidity problem changes the potential diversification benefits of investment in cryptocurrencies.

The remainder of the paper is organized in the following way. Section 2 describes data 
collection and methodology. Section 3 reports the empirical results and finally section 4 concludes.

\section{Data and methodology}

We choose the 10 most popular exchange traded-funds (hereafter, ETFs) from major asset classes and the 10 largest cryptocurrencies with respect to market capitalization. Our data period is from 5 days before China forbidding ICOs on the $4^{\text {th }}$ September 2017, until 5 days after the event. We collect trading prices, trading volume and market capitalization of cryptocurrencies from coinmarketcap.com. Our quoted price information is from Kaiko.com, and all of our data is specified in USD. Following Briere et al. (2015), we exclude short positions in order to obtain realistic investment possibilities.

We measure liquidity using the following methodologies:

$$
E S_{i, t}=2\left|P_{i, t}-M_{i, t}\right|
$$

Where $E S_{i, t}$ refers to the effective bid-ask spread at time period $t, P_{i, t}$ is the trading price at time period $t$ and $M_{i, t}$ is the midpoint between the bid and the ask price at time period $t$.

We also compute the two most popular price impact ratios for robustness. These are the Amihud (2002) RtoV ratio and the Florackis et al. (2011) RtoTR ratio. The RtoV ratio is defined as absolute daily return divided by daily monetary volume averaged over a trading period.

$$
\operatorname{RtoV}_{i}=\frac{1}{D_{i}} \sum_{d=1}^{D_{i}} \frac{\left|R_{i, d}\right|}{V_{i, d}}
$$

Where $\mid R_{i, d}$ land $V_{i, d}$ refers to the absolute return and monetary volume on day $d$ respectively, $D_{i}$ is the number of trading days. Florackis et al. (2011) indicate that the Rto $V$ ratio carries a significant size bias, given that it is highly positively correlated with market capitalization. Due to this shortcomings, Florackis et al. (2011) construct a new illiquidity ratio (RtoTR ratio), defined as the average ratio of daily absolute return to its turnover ratio: 


$$
\operatorname{RtoTR}_{i}=\frac{1}{\mathrm{D}_{i}} \sum_{d=1}^{\mathrm{D}_{i}} \frac{\left|\mathrm{R}_{i, d}\right|}{\mathrm{TR}_{i, d}}
$$

Where $T R_{i, d}$ refers to the turnover ratio on day $d, D_{i}$ and $R_{i, d}$ are the same as previously defined in equation (2).

Following Lo et al. (2003), the liquidity $(l)$ of portfolio $p$ can be defined as the weighted average of the liquidity of the securities in the portfolio:

$$
l_{p t} \equiv \sum_{i=1}^{n} w_{p i} l_{i t}
$$

Where $l_{\mathrm{pt}}$ represents one of our three liquidity measures for portfolio $p$ at time $t . w$ is the vector of portfolio weights, $w_{p i} \equiv\left[w_{p 1} w_{p 2} \ldots w_{p n}\right]$.

According to Lo et al. (2003), liquidity can be incorporated into the mean-variance portfolio optimization process by including the liquidity metric in the following objective function:

$$
\begin{gathered}
\operatorname{Max}_{\{w\}} w^{\prime} \mu-\frac{\lambda}{2} w^{\prime \Sigma w}+\phi w^{\prime} l_{t} \\
\text { Subject to } 1=w^{\prime} 1_{n}, w \geq 0
\end{gathered}
$$

Where $\mu$ is the vector of returns of securities, $\sum$ is the return covariance matrix of securities, $\lambda$ is the risk tolerance parameter, and $\phi$ is the weight placed on liquidity. We constrain $w$ to be non-negative so as to simplify the expression for the liquidity of $p$. We also include the Sharpe ratio as a portfolio performance measure, defined as the average return earned in excess of the risk-free rate per unit of risk.

\section{Results}

Our results reported in Table 1 display the portfolio performance before and after China forbidding ICOs. Panel A represents $\phi=0$, implying no weight for liquidity in the portfolio performance. Panel B shows the result of $\phi>0, \phi=0.1,0.2 \ldots 0.5$ suggesting a $10 \%, 20 \% \ldots .50 \%$ 
weight for liquidity in the portfolio performance. We measure 5 days before (after) the announcement, the liquidity measured by the effective spread, RtoV and RtoTR ratio ${ }^{1}$. Comparing the results between only ETFs $(E)$, ETFs and cryptocurrencies combined $(E+C)$, we observe that when $\phi$ equals 0 , including a portion of cryptocurrencies can dramatically increase the return of portfolio, from 0.067 to 0.285 . On the other hand, the standard deviation of return also increases from 0.040 to 0.118 due to the high volatility of cryptocurrencies. This is consistent with previous studies (see among others, Corbet et al., 2008), the high volatility of investment in cryptocurrencies is compensated by higher returns of portfolios. After the news announcement, the cryptocurrency market shows a negative reaction, implying the diversification benefits of cryptocurrencies disappear. When $\phi=0$, the return of $E$ and $E+C$ is 0.074 and 0.109 respectively, the difference is much smaller than before the event. However, the standard deviation between the two groups is even larger, 0.048 and 0.144 respectively.

In Panel $\mathrm{B}$, when we include liquidity in the portfolio $(\phi>0)$, the return is much lower than $\phi=0$ for both $E$ and $E+C$ investment portfolios. For example, in the effective spread group before the event, when $\phi$ increases from 0.1 to 0.5 , the return decreases from 0.066 to 0.036 for $E, 0.276$ to 0.201 for $E+C$. The standard deviation increases from 0.039 to 0.057 for $E$ and 0.120 to 0.141 for $E+C$. The Sharpe ratio of $E(E+C)$ also declines from $0.310(0.517)$ when $\phi=0.1$, to $0.245(0.325)$ when $\phi=0.5$. After the event announcement, both $E$ and $E+C$ returns are decreasing in a similar manner to Panel A. For instance, looking at the RtoV measure, the $E+C$ investment combination no longer provides any diversification benefits. This is because they have a lower Sharpe ratio than $E^{2}$

\section{[INSERT TABLE ONE HERE]}

\footnotetext{
${ }^{1}$ We also tried alternative event window and the results remain intact.

${ }^{2}$ For robustness, we repeat the econometric analysis for the largest 50 cryptocurrencies. The results (not reported) are quantitatively similar and are available from the authors upon request.
} 


\section{Conclusion}

In this paper, we observe that cryptocurrencies provide investment diversification before China banned ICOs in 2017. However, once we corporate liquidity into the portfolios, the gains from diversifying using cryptocurrencies is eliminated. Our study helps individual decision-making when cryptocurrencies and liquidity are included in investment portfolios.

\section{References}

Amihud, Y., (2002), "Illiquidity and stock returns: Cross-section and time-series effects", Journal of Financial Markets, Vol.5, pp, 31-56.

Baek, C., and Elbeck, M., (2015), "Bitcoins as an investment or speculative vehicle? A first look", Applied Economics letters, Vol.22(1), pp.30-34.

Briere, M., Oosterlinck, K., and Szafarz, A., (2015), "Virtual currency, tangible return: Portfolio diversification with Bitcoin", Journal of Asset Management, Vol.16(6), pp. 365-373.

Bouri, E., Molnár, P., Azzi, G., and Roubaud, D., (2017), "On the hedge and safe haven properties of Bitcoin: Is it really more than a diversifier?", Finance Research Letters, Vol.20, pp.192-198.

Corbet, S., Meegan, A., Larkin, C., Lucey, B., and Yarovaya, L.,(2018), "Exploring the dynamic relationships between cryptocurrencies and other financial assets", Economics Letters, Vol.165, pp.28-34.

Florackis,C., Gregoriou,A., and Kostakis,A., (2011)., "Trading frequency and asset pricing on the London Stock Exchange: Evidence firm a new price impact ratio", Journal of Banking \& Finance, Vol.35(12), pp.3335-3350.

Gregoriou, A., (2019), "Cryptocurrencies and asset pricing", Applied Economics Letters, Vol.26,(12), pp.995-998.

Guesmi, K., Samir, S., Ilyes, A., and Zied, F., (2019), "Portfolio diversification with virtual currency: Evidence from Bitcoin", International Review of Financial Analysis, Vol.63(C), pp.431-437.

Nakamoto,S., (2008), “Bitcoin: A peer-to-peer electronic cash system”, www. Bitccoi.org.

Lo, A.W., and Petrov, C., (2003), "It's 11pm-Do you know where your liquidity is? The meanvariance-liquidity frontier", Journal of Investment Management, Vol.(1), pp.55-93.

Platanakis, E., Sutcliffe, C. and Urquhart, A., (2018), "Optimal vs naïve diversification in cryptocurrencies”, Economics Letters, Vol 171, pp.93-96.

Platanakis, E. and Urquhart, A., (2019), "Portfolio management with cryptocurrencies: The role of estimation risk", Economics Letters, Vol 177, pp.76-80. 
Platanakis, E. and Urquhart, A., (2019), "Should investors include Bitcoin in their portfolios? A portfolio theory approach", The British Accounting Review, p.100837.

Zhang, S. and Gregoriou, A., (2020), "The price and liquidity impact of China forbidding initial coin offerings on the cryptocurrency market", Applied Economics Letters, pp.1-4. 
Table 1: Portfolio performance and liquidity of cryptocurrencies around China banning ICOs

Table 1 reports the mean of return $(R)$, standard deviation of return $(S T D)$ and Sharpe ratio of portfolio $\rho$ which includes only ETF $(E)$ and both ETF and cryptocurrencies $(E+C), 11$-days around the banning initial coin offerings (ICOs) announcement period. $\phi$ is the liquidity parameter. The liquidity measures are effective spread, RtoV and RtoTR ratios.

Panel A: $\phi=0$

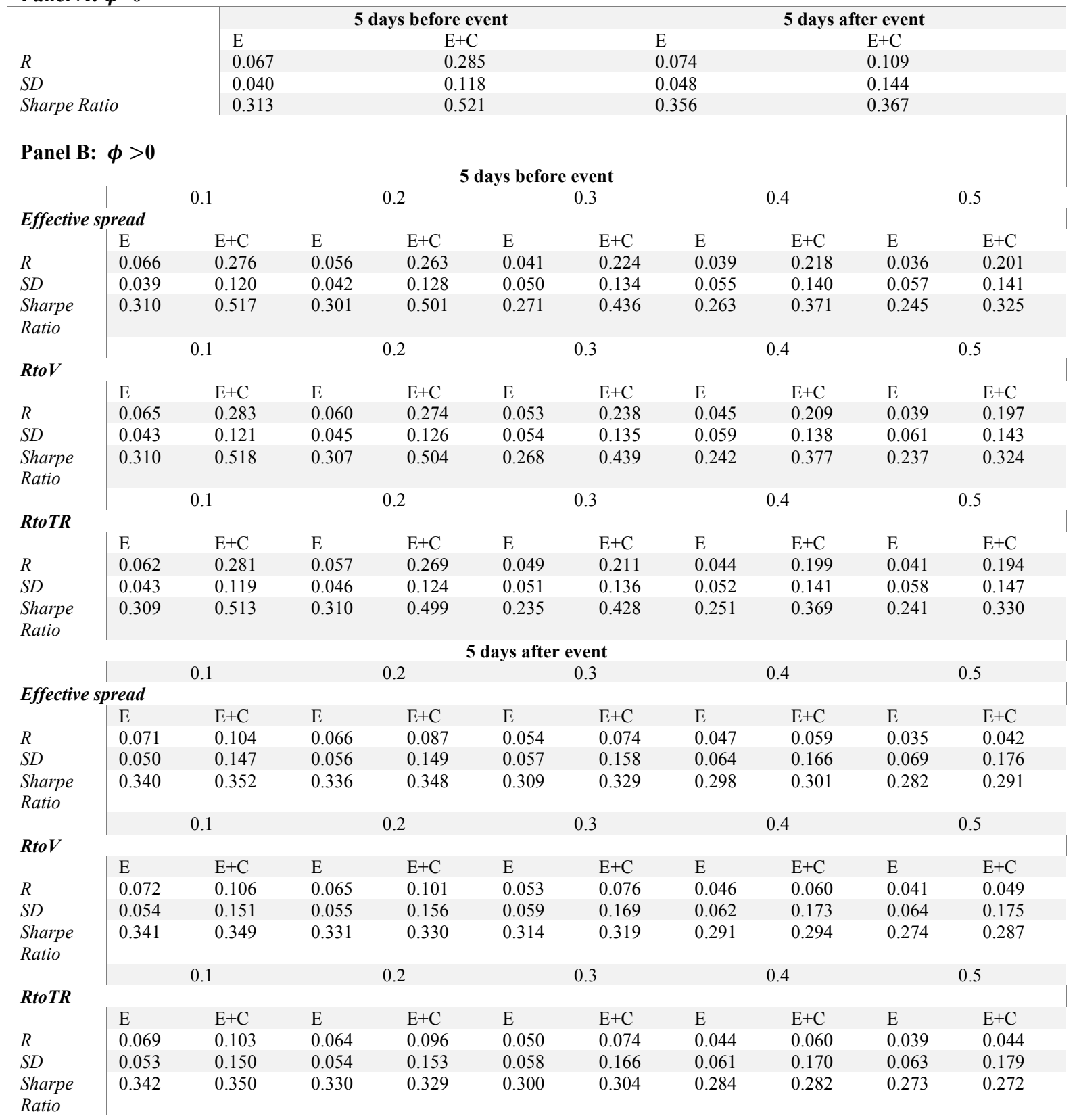

